\title{
Brightness of the solar $F$-corona
}

\author{
Hiroshi Kimura and Ingrid Mann \\ Max-Planck-Institut für Aeronomie, Max-Planck-Straße 2, D-37191 Katlenburg-Lindau, Germany
}

(Received October 7, 1997; Revised March 18, 1998; Accepted March 25, 1998)

\begin{abstract}
We discuss our present knowledge about the brightness of the solar $F$-corona in the wavelength range from the visible to the middle infrared. From the general trend of the observational data, the $F$-corona is regarded as the continuous extension of the zodiacal light at smaller elongation of the line of sight. A contribution of thermal emission from dust is indicated by the increasing $F$-coronal brightness in comparison to the solar spectrum towards longer wavelength. As compared with the $F$-coronal brightness, the polarization and color in the visible regime are not well determined due to the high sensitivity of these quantities to the observational accuracy. Aside from observational problems, our present interpretation of the $F$-coronal brightness is also limited due to ambiguities in the inversion of the line of sight integral. Nevertheless, the measurements and model calculations of the brightness can be used to deduce some physical properties of dust grains. We show that the hump of the near-infrared brightness at 4 solar radii, which was sometimes observed in the corona, is related rather to the physical properties of dust grains along the line of sight than to the existence of a dust ring as previously discussed. We also show that the appearance or disappearance of the near-infrared peak in the coronal brightness cannot be described in any periodic cycle for each wavelength range.
\end{abstract}

\section{Introduction}

The brightness of the solar corona has long been known to consist of mainly two components, i.e., $K$ - and $F$-corona, although, as a further component, emission lines of highly charged coronal ions also influence the coronal brightness. The $K$ component follows the continuous slope of the solar spectrum, called the "kontinuierliche Korona", and its brightness is produced by Thomson scattering of sunlight in an atmosphere of free electrons surrounding the Sun. The $F$ component shows the Fraunhofer lines of the solar spectrum and nowadays is accepted as sunlight scattered by interplanetary dust particles (IDPs), distributed in interplanetary space along the line of sight (LOS). The contribution of thermal emission ( $T$ component) to the total brightness of the solar corona increases with increasing wavelength and hence the $T$ component can dominate the brightness of the $F$-corona in the near-infrared wavelength range (Peterson, 1963; Mann, 1992).

In the classical approach, the solar corona has been studied on the basis of its brightness, polarization, and color mainly in the visible wavelengths (see Blackwell et al., 1967). Since the sixties, further information has been obtained from nearinfrared observations (Peterson, 1967, 1969; MacQueen, 1968), and with infrared imaging systems in the nineties (Hodapp et al., 1992; Kuhn et al., 1994; Tollestrup et al., 1994).

In this paper, we shall take a general view of the $F$-coronal brightness in the wavelength range from the visible to the infrared, as well as the polarization and color measured over more than thirty years. Moreover, we discuss some attempts

Copy right (C) The Society of Geomagnetism and Earth, Planetary and Space Sciences (SGEPSS); The Seismological Society of Japan; The Volcanological Society of Japan; The Geodetic Society of Japan; The Japanese Society for Planetary Sciences. for models of the $F$-coronal brightness and the possibilities of variable features in the near-infrared brightness.

\section{F-Corona from Zodiacal Light}

The visible brightness of the solar corona is difficult to observe in daylight, because the brightness of the day sky exceeds the coronal brightness except at the limb of the Sun (see Fig. 1). Consequently, the brightness of the solar corona is observed either during total solar eclipses when the sky brightness decreases by three orders of magnitude or from spaceborne externally occulted coronagraphs. Since the brightness of the $K$-corona considerably decreases with increasing elongation, the $F$-corona dominates the brightness of the solar corona at elongations greater than 4 solar radii $\left(R_{\odot}\right)$. Observations of the outermost solar corona, in other words, the inner zodiacal light have been performed from space to avoid atmospheric contamination (MacQueen et al., 1973; Leinert et al., 1974) and have proven that the visible solar corona is smoothly connected to the zodiacal light at small elongations of the LOS. Improved white light observations may be available in the near future with the measurements of the coronagraphs on the SOHO satellite (see Brueckner et al., 1995). It is worthwhile noting, however, that infrared observations of the zodiacal light have been made mainly at wavelength $10 \mu \mathrm{m}$ and beyond, usually for elongations more than 60 degree from the Sun (see Hanner (1991) for a review). Accordingly, there is still an observational gap in the near-infrared brightness between the solar corona, which so far is mainly known from eclipse observations, and the zodiacal light, which has been observed in this wavelength range recently (see Matsumoto et al. (1996) for the most recent review).

The properties of the zodiacal light are related to the spatial distribution and physical properties of IDPs (see the most 


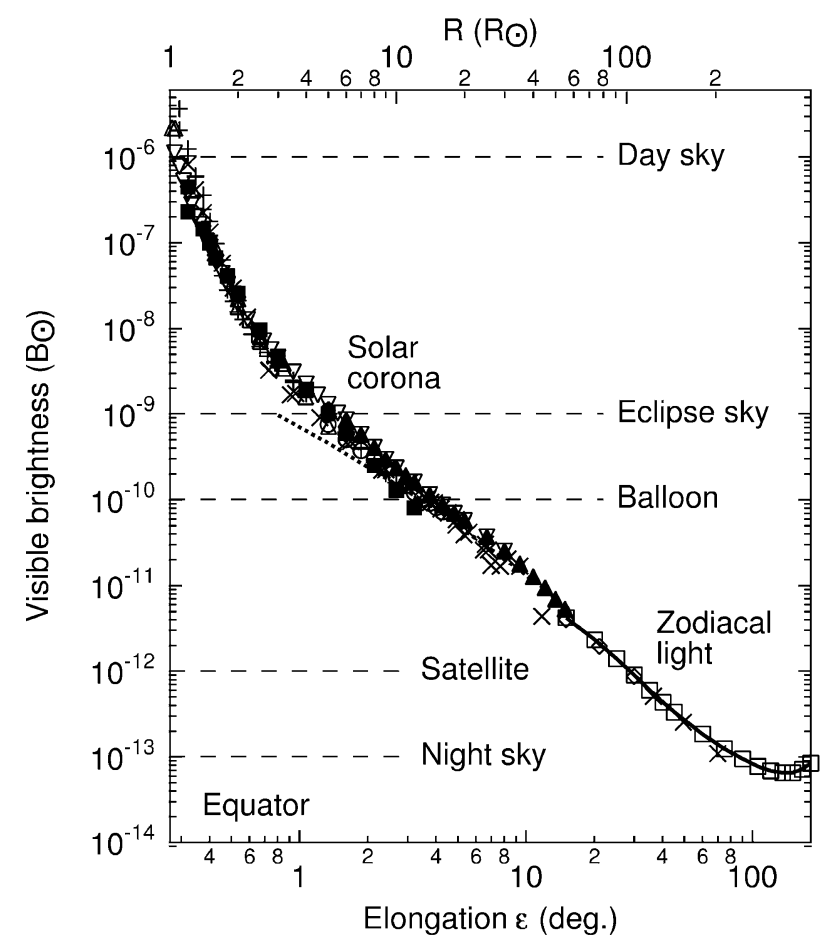

Fig. 1. The visible brightness of the solar corona in the equatorial plane of the Sun as a function of elongation. Observations: Blackwell (1955); Allen (1956); Waldmeier (1961); Gillett et al. (1964); Blackwell and Petford (1966a); MacQueen et al. (1973); Leinert et al. (1974); Saito et al. (1977); Dürst (1982); Nikolsky et al. (1983); Levasseur-Regourd (1996).

recent review of Mann (1998) for further discussion). In the same manner, the brightness of the $F$-corona depends on the spatial and size distribution of IDPs along the LOS as well as on their light-scattering and thermal properties. Since the main contribution to the zodiacal light arises from IDPs large compared to the wavelength of the scattered light (Giese et al., 1978), the average scattering property of IDPs shows a strong component of light scattered in the forward direction with respect to the propagation of incident sunlight. This is referred to as the diffraction peak in the limit of large particles. The brightness produced from dust near the observer stems from the forward scattering regime, whereas the contribution of near-solar dust particles on the visible brightness of the $F$-corona (integrated along the LOS) comes from sunlight scattered at phase angles close to $90^{\circ}$. Consequently, the scattered sunlight by far-solar dust particles obscures the direct detection of the near-solar dust particles in the coronal brightness. In fact, visible observations of the solar $F$ corona have not shown any feature of a dust-free zone, which is expected to be present as a result of sublimation of dust particles near the Sun (Russell, 1929). Accordingly, it may not be straightforward to investigate the innermost of the zodiacal dust cloud in terms of the $F$-coronal brightness. Nevertheless, we expect that physical properties of IDPs in the inner solar system, which are not well described so far, can be derived from the properties of the $F$-corona and make its further study worthwhile. As far as the infrared brightness of the solar corona is concerned, information about the geome- try of the dust cloud near the Sun could be derived from the $T$ component that arises mainly from near-solar dust grains. Also observations of a feature in the near-infrared brightness have caused a discussion of dust rings near the Sun, although some of the later observations could not detect such a feature. A detailed discussion will be given later through the paper.

\section{Polarization of the $\boldsymbol{F}$-Corona}

The linear polarization of the total $K+F$ corona tends to decrease with increasing elongation within the corona and then to increase again into the zodiacal light. However, from the 1983 solar eclipse measurement, Isobe et al. $(1985,1987)$ derived an enhancement of the coronal polarization between 4 and $5 R_{\odot}$, which was concluded to be caused by scattered light of near-solar dust grains at phase angle $\theta=90^{\circ}$. A recent observation of the 1991 eclipse has shown no hump in the linear polarization between 3 and $6.4 R_{\odot}$ within an upper limit of $10 \%$ for the excess polarization (Tanabé et al., 1992). Although we cannot disprove the excess polarization in the 1983 measurement without further observations, the more recent observation implies that the scattered sunlight from near-solar dust particles at phase angle $\theta \simeq 90^{\circ}$ yields a minor contribution to the total polarization.

The polarization of the $F$-corona can be derived from separation of the $K$ - and $F$-corona by a simultaneous measurement of the total $K+F$ coronal brightness and polarization and of the depth of Fraunhofer lines. Blackwell and Petford (1966b) obtained the polarization of the $F$-corona to be almost zero at elongations ranging from 10 to $16 R_{\odot}$, although two independent measurements at different eclipses of 1954 and 1963 were used for separation of the $K$ - and $F$-corona. Blackwell et al. (1967) have modeled the $F$-coronal polarization from 5 to $16 R_{\odot}$ at visible wavelength so that the model polarization can smoothly connect the polarization data of Blackwell and Petford (1966b). Assuming the $K$-coronal polarization to be constant beyond $5 R_{\odot}$, Koutchmy and Lamy (1985) also have established a model of the $F$-coronal polarization, but there is a large difference by order of magnitude between the two models mentioned above. In general, it should be emphasized that small uncertainties in the determination of the strongly polarized $K$-coronal brightness have a drastic influence on the derived $F$-coronal polarization.

Mann (1992) has attempted to derive the local properties of near-solar dust particles from the model of Blackwell et al. (1967) and has concluded that the local polarization of the near-solar dust grains located at heliocentric distances smaller than $r=0.1$ AU has a radial slope between $r^{2.8}$ and $r^{2.6}$ depending on model assumptions. On the other hand, the local polarization of the zodiacal light at visible wavelength has been derived by the nodes of lesser uncertainty method (see Levasseur-Regourd (1996) for a review). The local polarization of IDPs at constant phase angle $\theta=90^{\circ}$ varies as $r^{0.5 \pm 0.1}$ between 0.5 and $1.4 \mathrm{AU}$ from the Sun with a total value of $0.3 \pm 0.03$ at $1 \mathrm{AU}$. The local polarization at $\theta=90^{\circ}$ steeply decreases below $r=0.3 \mathrm{AU}$, which is continued into the $F$-corona and may suggest a drastic change in the physical properties of IDPs near the Sun (Levasseur-Regourd et al., 1991; Mann, 1991). Further analysis, however, has shown that the derived polarization properties of dust near the Sun are not compatible with the present understanding of single 
particles scattering properties (Mann, 1993a). We conclude that the $F$-coronal polarization is not very well understood so far and that a further analysis needs new and better observations combined with a careful separation of the $K$ - and $F$-coronal brightness.

\section{Shape of the $\boldsymbol{F}$-Corona}

The separation of the $K$ - and $F$-corona in the total brightness enables us to investigate the shape of the $F$-corona along the elongation of the LOS. A model of the $F$-corona given by Koutchmy and Lamy (1985) describes the profile of the zodiacal light and $F$-coronal brightness as $\varepsilon^{-2.25}$ along the equator and $\varepsilon^{-2.47}$ along the pole beyond $4 R_{\odot}$ from the Sun, based on their compilation of $F$-corona and zodiacal light data at visible wavelength range. However, due to the different conditions of forward scattering in the $F$-coronal brightness, we cannot expect the zodiacal light and the $F$ coronal brightness to be described with the same slope of elongations.

Recent discussions of the IAU commission 21 assume a radial slope of the $F$-coronal brightness of $r^{-2.5}$ in the equatorial plane and $r^{-2.8}$ in the direction of the solar poles (Leinert et al., 1998). On the other hand, the radial slope of the near-infrared $F$-coronal brightness can be derived from observations of the 1991 eclipse, where the measurements with imaging detectors could give a relatively reliable description of the brightness slopes, although the sky conditions were not really good (see, e.g., Hodapp et al., 1992). The equatorial and polar brightness of the near-infrared $F$-corona were described as $B_{\text {eq }} \sim r^{-\left(1.9_{-0.1}^{+0.2}\right)}$ and $B_{\text {pol }} \sim r^{-(2.3 \pm 0.2)}$, respectively, for regions inside $8 R_{\odot}$ (MacQueen and Greeley, 1995). We conclude that the shape of the $F$-corona tends to be more spherical than the zodiacal light and that a spectral variation of its shape may result from the influence of the $T$ components at larger wavelength.

\section{Color of the $\boldsymbol{F}$-Corona}

Figure 2 shows the wavelength dependence of the coronal brightness in units of the mean solar disk brightness $B_{\odot}$. The brightness data are obtained at an elongation of $4 R_{\odot}$ along the ecliptic plane and the references are given in the figure caption. A dashed line indicates the color of the zodiacal light extrapolated to $4 R_{\odot}$ according to the dependence of color on elongation derived from the Helios photometer measurements (cf. Leinert et al., 1982). Because the Thomson scattering of coronal free electrons shows neutral color, deviations of the coronal brightness from the straight line parallel to the horizontal axis indicate the color of the $F$-corona. Concerning the visible wavelength range, the large uncertainty in the absolute observed brightness prevents us from directly comparing the color of the solar corona to that of the zodiacal light extrapolated to the elongation of $4 R_{\odot}$, whereas Koutchmy and Lamy (1985) have concluded a strong reddening in the $F$-corona. In the near-infrared regime, the color of the solar corona is significantly redder than that of the Sun, although the available data are scattered in magnitude. As was shown by Mann (1993b), a reddening of the solar corona in the near-infrared wavelength could arise from a thermal emission component as well as from the influence of the forward scattering of particles far from the Sun (see

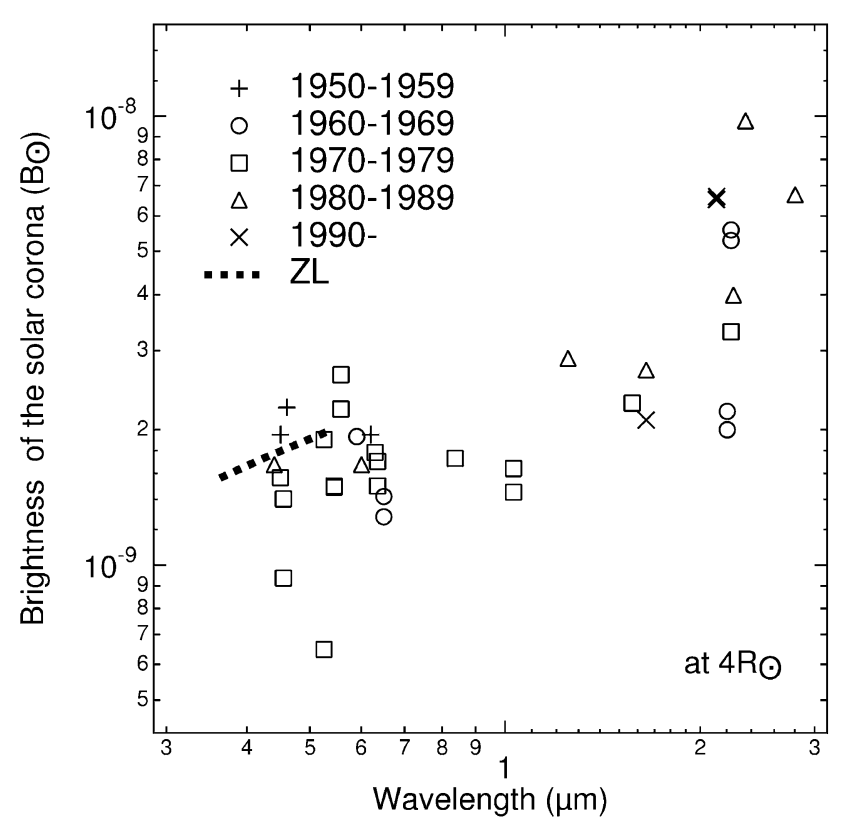

Fig. 2. The brightness of the solar corona at an elongation of $4 R_{\odot}$ in the unit of solar brightness from visible to near-infrared wavelength describes the color of the solar corona. Observations: Allen (1956); Waldmeier (1961); MacQueen (1968); Peterson (1969, 1971); Koomen et al. (1970); Newkirk et al. (1970); Pepin (1970); MacQueen et al. (1973); Smartt et al. (1974); Saito et al. (1977); Ajmanov and Nikolsky (1980); Rao et al. (1981); Dürst (1982); Mampaso et al. (1983); Nikolsky et al. (1983); Maihara et al. (1985); Hodapp et al. (1992); Tollestrup et al. (1994).

van de Hulst (1947) for the diffraction reddening). Measurements with high accuracy of the solar corona at visible and near-infrared wavelengths, which show the dependence of the reddening on the elongation of the LOS, would provide useful information on not only the edge of dust-free zone, but also the size distribution of IDPs along the LOS (Mann, 1993b; MacQueen et al., 1996).

\section{The Configuration of the Dust Cloud near the Sun}

Theoretical investigation of the sublimation of the hot near-solar dust has indicated that the edge of the dust-free zone for spherical quartz grains is located about $4 R_{\odot}$ from the Sun (Over, 1958). The effect of the dust-free zone on thermal emission brightness has been supposed to cause the sharp break of the brightness at the edge of the dust-free zone (Peterson, 1963). As might have been expected, Peterson (1967, 1969) and MacQueen (1968), independently, detected a sharp peak in the near-infrared brightness of the solar corona at $4 R_{\odot}$ from the Sun during the total solar eclipse of 1966. Because the near-infrared peak appeared simultaneously at the east and the west side of the Sun, Peterson (1967, 1969) concluded that the near-solar dust grains move in circular orbit. Based on a balloon-borne observation of the 1983 eclipse, Mizutani et al. (1984) have suggested the concentration of the near-solar dust cloud towards the ecliptic plane in order to explain a detected feature in the east-west side slope of the brightness. According to these observations, the near-solar dust cloud can be assumed to be symmetric with respect to the Sun and be distributed near the ecliptic plane. 
The near-solar dust grains circularly orbiting near the ecliptic plane seem to be a natural consequence of the PoyntingRobertson effect on IDPs, because the Poynting-Robertson effect diminishes simultaneously the orbital eccentricity and semimajor axis of IDPs whose orbital inclinations are between $\pm 30^{\circ}$ for most of the zodiacal cloud models (see Mann and Grün, 1992). This in turn suggests the correlation of the near-solar dust cloud to the zodiacal cloud.

On the basis of theoretical studies concerning the orbital evolution of circumsolar dust grains, Belton $(1966,1967)$ has found that the orbital evolution of dust particles is stabilized at the edge of the dust-free zone. Subsequently, Mukai and Yamamoto (1979) have constructed a model of the near-solar dust rings consisting of spherical silicate and carbon grains to explain the observed infrared brightness. Hence, the sharp peak detected in the near-infrared brightness at $4 R_{\odot}$ has been interpreted as a result of the solar dust ring. Kimura et al. (1997), however, have shown that highly fluffy porous grains may not produce a remarkable ring structure around the Sun. On the one hand, Mann and MacQueen (1993) have proposed a flat radial distribution of dust number density in the vicinity of the Sun in order to explain the $F$-coronal brightness deduced from near-infrared measurement during the 1991 solar eclipse by Hodapp et al. (1992). Such a distribution may result from the gradual subsequent sublimation of particles of different material compositions at different distances from the Sun (see Mann et al., 1994). Consequently, further analysis of dust dynamics near the Sun may be required to obtain further information on the configuration of near-solar dust cloud.

Furthermore, Kimura et al. (1998) have shown that a low ratio of carbon to silicate porous grains near the Sun results in the absence of the sharp peak in the near-infrared $F$-coronal brightness even if the solar dust ring exists. Therefore, the appearance of the peak feature in the near-infrared $F$-coronal brightness depends not only on the configuration of the dust clouds but also on the physical properties of the near-solar dust particles. At this point, both theoretical models and observations cannot give a final explanation to the topic of dust rings as well as brightness features.

\section{Thermal Properties of Near-Solar Dust}

The $T$ component of the coronal brightness can provide information about thermal properties of near-solar dust as well as the geometry of the dust cloud near the Sun. Multiwavelength measurements of the thermal emission brightness yield the color temperature of dust grains. Peterson (1971) has derived the color temperature of $2160 \pm 200 \mathrm{~K}$ for nearsolar dust from multi-wavelength measurement during the total solar eclipse in 1970 and has suggested the presence of carbon (graphite) grains close to the sublimation zone. Mukai and Mukai (1973) have shown from model calculations that the equilibrium temperature of spherical carbon grains at $4 R_{\odot}$ can explain the 1970 eclipse observation. On the other hand, the analysis of multi-wavelength measurements during the 1983 total solar eclipse led to the conclusion that the enhanced brightness at $4 R_{\odot}$ originates from thermal emission of large silicate (olivine) particles near the Sun (Mizutani et al., 1984; Maihara et al., 1985). Mann and MacQueen (1993) have derived from the 1991 obser- vation by Hodapp et al. (1992) that an average temperature of IDPs is about $10 \%$ below the blackbody temperature and this was later shown to be consistent with the existence of silicate fluffy porous particles, which contain impurities of absorbing material (Mann et al., 1994).

The existence of silicate mineral in IDPs is evident from detailed laboratory analysis of IDPs collected from the upper atmosphere of the Earth (e.g., Brownlee et al., 1980) and from the detection of the silicate $10 \mu \mathrm{m}$ emission feature in comets that are one of the source of IDPs (e.g., Hanner et al., 1994). However, there is no direct proof for the existence of silicate particles near the Sun, such as the observation of a silicate feature in the $T$ component of the coronal brightness. The mid-infrared brightness of the solar corona has been measured only twice during the total solar eclipses of 1970 (Mankin et al., 1974) and 1973 (Léna et al., 1974). The existence of silicate particles might be suggested by these mid-infrared observations, since the brightness was considerably higher than the extrapolation from the visible brightness based on the assumption of blackbody radiation for the dust emission. The ground-based observation made by Mankin et al. (1974), however, was highly contaminated by sky noise, as pointed out by the authors and therefore the $10 \mu \mathrm{m}$ brightness of the 1970 eclipse must be regarded as only the upper limit of mid-infrared coronal radiance. Although the measurement by Léna et al. (1974) was carried out from a supersonic aircraft to decrease atmospheric noise, the observed brightness exceeded the upper limit determined by Mankin et al. (1974). This presumably implies that the aircraft measurement during the 1973 eclipse was contaminated by the apparatus, e.g., a window in the roof of the aircraft. Accordingly, the present observational data in the mid-infrared wavelength range do not allow the derivation of the material composition of near-solar dust particles.

\section{Disappearance of the Excess Emission in the Near-Infrared Brightness}

Observations of the near-infrared brightness have shown that the peak feature around $4 R_{\odot}$ from the Sun has not always been detected. Therefore, the excessive emission of the near-infrared brightness has been regarded as a temporal feature and thus several explanations have been suggested for a temporal variation in the near-infrared brightness of the $F$-corona.

Hodapp et al. (1992) and MacQueen et al. (1994) have proposed that a transient ring of near-solar grains can occasionally be supplied by the Kreutz group of sungrazing comets, because of their episodic appearance (see MacQueen and St. Cyr, 1991). As noted by Hodapp et al. (1992), however, it is very difficult to supply the dust into the ecliptic plane from Kreutz sungrazers, which are in orbits with high inclination. In addition, high eccentricity of the sungrazing comets prevents the cometary dust from staying in a bound orbit around the Sun and the supply of cometary dust from the sungrazer is a very local phenomenon that is inconsistent with the symmetrical feature of the near-infrared hump around $4 R_{\odot}$ on the both side of the Sun (cf. Peterson, 1967, 1969). Although the cometary dust grains temporarily increase the coronal brightness (Michels et al., 1982), the observable time is too short to explain the excess emission of near-infrared brightness 
detected over several months (cf. MacQueen, 1968).

On the other hand, Tanabé et al. (1992), Isobe and Satheesh-Kumar (1993a, 1993b), and C. (1995) have concluded that the temporal variation in the near-infrared brightness correlates with the solar activity cycle and therefore can be explained by the dissipation of the solar dust ring at maximum phase of the solar cycle by virtue of the Lorentz force acting on small charged grains under the strong solar magnetic field. As pointed out by Mann (1993a), however, the Lorentz force can only influence the spatial distribution of the small near-solar dust grains, which are unlikely to produce a significant part of the coronal brightness. Recently, the correlation between the solar activity cycle and the detection of the near-infrared peak in the solar $F$-corona has been rejected by Krivov et al. (1998a, 1998b) based on the dynamical behavior of circumsolar charged grains in the time-dependent solar magnetic field. Furthermore, the explanation for the time-variable feature of the near-infrared brightness in terms of the solar activity cycle requires to ignore artificially several observational reports, such as a detection of the peak at $4 R_{\odot}$ in the K-band (a wavelength of $2.2 \mu \mathrm{m}$ ) brightness during the total solar eclipse of 1970 (cf. Peterson, 1971) and no feature in the brightness at the same wavelength during the 1983 solar eclipse (cf. Mizutani et al., 1984; Maihara et al., 1985). Looking at observational results with care, it may be noticed that the 1983 eclipse measurement has shown the peak feature of $4 R_{\odot}$ clearly at a wavelength of $1.65 \mu \mathrm{m}$ and

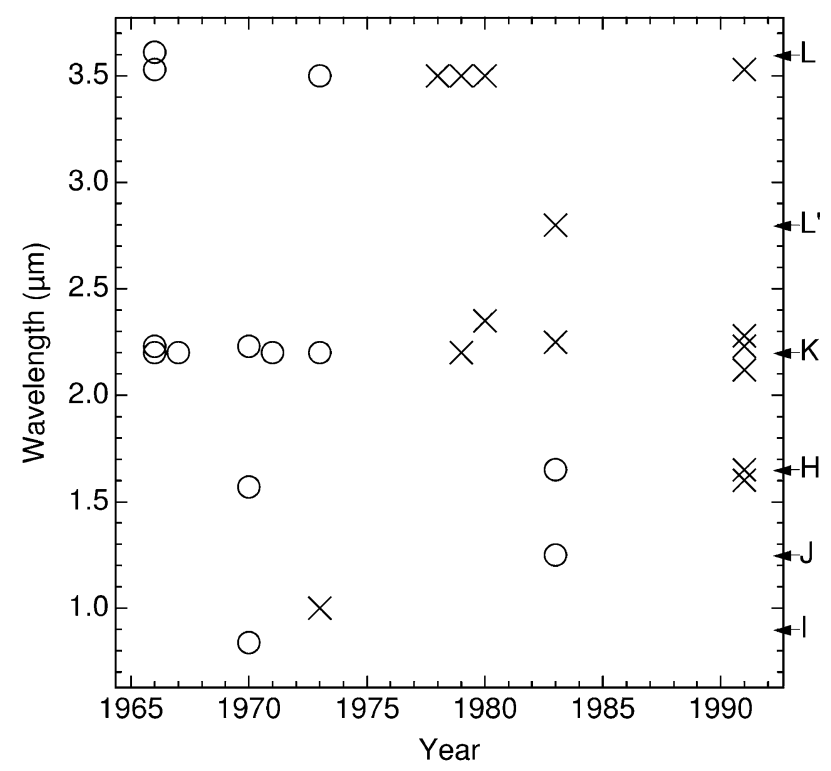

Fig. 3. The peak feature around $4 R_{\odot}$ from the sun was detected (circles) at each wavelength range and then disappeared (crosses). I-band: Peterson (1971); Smartt et al. (1974). J-band: Mizutani et al. (1984); Maihara et al. (1985). H-band: Peterson (1971); Mizutani et al. (1984); Maihara et al. (1985); Lamy et al. (1992); Kuhn et al. (1994); Tollestrup et al. (1994). K-band: Peterson (1967, 1969, 1971); MacQueen (1968); Adney (1973); Strong (1974); Rao et al. (1981); Mampaso et al. (1982, 1983); Mizutani et al. (1984); Maihara et al. (1985); Hodapp et al. (1992); Lamy et al. (1992); Skomorovsky et al. (1992); Kuhn et al. (1994); MacQueen et al. (1994). L'-band: Mizutani et al. (1984); Maihara et al. (1985). L-band: Peterson (1967, 1969); Strong (1974); Ney (1980, private communication to Mampaso et al. (1983)); Mampaso et al. (1982, 1983); Skomorovsky et al. (1992). weak enhancement without definite peak at $1.25 \mu \mathrm{m}$, but the absence of any hump at other wavelengths (Mizutani et al., 1984; Maihara et al., 1985). This implies that we must investigate near-infrared observations at different wavelength separately. In Fig. 3, we compile observational results concerning appearance (circles) and disappearance (crosses) of the near-infrared hump at different wavelength and different years of the observations. Note that we display the observational results purely according to the observer's report without any own judgments on the reliability. As far as the data in one wavelength band are concerned, the appearance and disappearance of the infrared hump in the $F$-coronal brightness around $4 R_{\odot}$ from the Sun does not show any cyclic variation. We can merely state from Fig. 3 that the excessive emission in the near-infrared brightness has appeared in the early observations since 1966 and disappeared in the late observations.

\section{Summary and Future}

Our present knowledge of the $F$-coronal brightness is still limited due to difficulties of the coronal observations, separation problems of the $K$ - and $F$-component in the corona, and contamination of instrumental and atmospheric stray light components. From the general trend of the data, the $F$ corona is the continuation of the zodiacal light at smaller elongation of the LOS. The $F$-coronal polarization and color in the visible regime are not accurately determined so far, but we can expect space experiments, such as those on SOHO to yield a better understanding in the near future. However, even with a reliable data set, the analysis of the near-solar dust cloud is difficult, mainly due to problems of the LOS inversion from the $F$-coronal brightness. At this point, further knowledge of the spectral slope of the coronal brightness especially of the near-infrared brightness would be helpful. In general, the infrared brightness of the $F$-corona may provide more information on the physical properties and spatial distribution of near-solar dust particles than the visible brightness does.

As far as the spatial distribution of dust near the Sun is concerned, dynamical effects may cause a dust ring around the Sun, which is often discussed to cause a peak feature in the thermal emission brightness. On the other hand, model calculations have also shown that the existence of the dust-free zone alone can yield a peak feature in the thermal emission brightness as well. However, its appearance in the coronal brightness depends on the material composition of the nearsolar dust particles, regardless of the existence of a solar dust ring around the Sun.

The spatial distribution of near-solar dust grains around the Sun is still the subject of debate from the available data and can only partly be derived from near-earth observations. Experiments on a mission to the inner solar system, such as a solar probe mission may provide further knowledge about the dust environment near the Sun. We have shown that the hump of the near-infrared brightness at $4 R_{\odot}$ has not appeared in any periodic cycle at each wavelength range. Nevertheless, the disappearance of the near-infrared feature is still an open question and raises the question of a temporal variation in the near-solar dust cloud. For a further exploration of this issue, long term observations may be necessary. 
Acknowledgments. We would like to thank James F. McKenzie, Alexander Krivov, Klaus Scherer, Peter Hillebrand, and Natalia Krivova for useful suggestions and discussions and Valery Dikarev for his kind help. We also thank the anonymous referee for useful comments. This work is partly supported by the Japan Society for the Promotion of Science (JSPS) and by the BMBF.

\section{References}

Adney, K. J., Reduction of data, balloon-borne infrared coronagraph flight of October 24, 1971, NASA Report, October 1973 (UMASS-ARF-73-284), cited by Strong (1974)

Ajmanov, A. K. and G. M. Nikolsky, The colour of the solar corona and dust grains in it, Solar Phys., 65, 171-179, 1980.

Allen, C. W., Coronal photometry at the eclipse of 1954 June 30, Mon. Not. Roy. Astron. Soc., 116, 69-76, 1956.

Belton, M. J. S., Dynamics of interplanetary dust, Science, 151, 35-44, 1966.

Belton, M. J. S., Dynamics of interplanetary dust particles near the Sun, in The Zodiacal Light and the Interplanetary Medium, edited by J. L. Weinberg (NASA SP-150), pp. 301-306, NASA, Washington, 1967.

Blackwell, D. E., A study of the outer corona from a high altitude aircraft at the eclipse of 1954 June 30, Mon. Not. Roy. Astron. Soc., 115, 629-649, 1955.

Blackwell, D. E. and A. D. Petford, Observations of the 1963 July 20 solar eclipse I. Spectroscopic separation of the $F$ and $K$ components of the solar corona at large distances from the Sun, Mon. Not. Roy. Astron. Soc., 131, 383-398, 1966a.

Blackwell, D. E. and A. D. Petford, Observations of the 1963 July 20 solar eclipse II. The electron density in the solar corona in the region $5<$ $R / R_{0}<16$ obtained from measurements of Fraunhofer line depth, and the polarization of the $F$ corona, Mon. Not. Roy. Astron. Soc., 131, 399 406, 1966b.

Blackwell, D. E., D. W. Dewhirst, and M. F. Ingham, The zodiacal light, Adv. Astron. Astrophys., 5, 1-69, 1967.

Brownlee, D. E., L. Pilachowski, E. Olszewski, and P. W. Hodge, Analysis of interplanetary dust collections, in Solid Particles in the Solar System, edited by I. Halliday and B. A. McIntosh, pp. 333-342, Reidel, Dordrecht, 1980.

Brueckner, G. E., R. A. Howard, M. J. Koomen, C. M. Korendyke, D. J. Michels, J. D. Moses, D. G. Socker, K. P. Dere, P. L. Lamy, A. Llebaria, M. V. Bout, R. Schwenn, G. M. Simnett, D. K. Bedford, and C. J. Eyles, The large angle spectroscopic coronagraph (LASCO) Visible light coronal imaging and spectroscopy, Solar Phys., 162, 357-402, 1995.

C., D. P., Variability of circumsolar dust ring, Solar Phys., 159, 181-190, 1995.

Dürst, J., Two colour photometry and polarimetry of the solar corona of 16 February 1980, Astron. Astrophys., 112, 241-250, 1982.

Giese, R. H., K. Weiss, R. H. Zerull, and T. Ono, Large fluffy particles: a possible explanation of the optical properties of interplanetary dust, Astron. Astrophys., 65, 265-272, 1978.

Gillett, F. C., W. A. Stein, and E. P. Ney, Observations of the solar corona from the limb of the Sun to the zodiacal light, July 20, 1963, Astrophys. J., 140, 292-305, 1964.

Hanner, M. S., The infrared zodiacal light, in Origin and Evolution of Interplanetary Dust, Astrophysics and Space Science Library, Vol. 173, edited by A. C. Levasseur-Regourd and H. Hasegawa, pp. 171-178, Kluwer, Dordrecht, 1991.

Hanner, M. S., D. K. Lynch, and R. W. Russell, The 8-13 micron spectra of comets and the composition of silicate grains, Astrophys. J., 425, 274$285,1994$.

Hodapp, K.-W., R. M. MacQueen, and D. N. B. Hall, A search during the 1991 solar eclipse for the infrared signature of circumsolar dust, Nature, 355, 707-710, 1992.

Isobe, S. and A. Satheesh-Kumar, An explanation for time dependent variability of the solar dust ring, Astrophys. Space Sci., 205, 297-303, 1993a.

Isobe, S. and A. Satheesh-Kumar, An effect of Lorentz force on interplanetary dust, in Meteroroids and Their Parent Bodies, edited by J. Štohl and I. P. Williams, pp. 381-385, Astronomical Inst. Slovak Acad. Sci., Bratislava, 1993b.

Isobe, S., T. Hirayama, N. Baba, and N. Miura, Optical polarization observations of circumsolar dust during the 1983 solar eclipse, Nature, 318, 644-646, 1985.

Isobe, S., T. Hirayama, N. Baba, and N. Miura, Optical coronal polarization and solar dust ring, Publ. Astron. Soc. Japan, 39, 667-677, 1987.

Kimura, H., H. Ishimoto, and T. Mukai, A study on solar dust ring formation based on fractal dust models, Astron. Astrophys., 326, 263-270, 1997. Kimura, H., I. Mann, and T. Mukai, Influence of dust shape and material composition on the solar F-corona, Planet. Space Sci., 1998 (in press).

Koomen, M. J., J. D. Purcell, and R. Tousey, Rocket observations of the corona on March 7, 1970, Nature, 226, 1135-1138, 1970.

Koutchmy, S. and P. L. Lamy, The $F$-corona and the circum-solar dust evidences and properties [G. Nikolsky memorial lecture], in Properties and Interactions of Interplanetary Dust, Astrophysics and Space Science Library, Vol. 119, edited by R. H. Giese and P. Lamy, pp. 63-74, Reidel, Dordrecht, 1985.

Krivov, A., I. Mann, and H. Kimura, The circumsolar dust complex and solar magnetic field, Earth Planets Space, 50, this issue, 551-554, 1998a.

Krivov, A., H. Kimura, and I. Mann, Dynamics of dust near the Sun, Icarus, 1998b (in press).

Kuhn, J. R., H. Lin, P. Lamy, S. Koutchmy, and R. N. Smartt, IR observations of the $K$ and $F$ corona during the 1991 eclipse, in Infrared Solar Physics, IAU Symp. No. 154, edited by D. M. Rabin, J. T. Jefferies, and C. Lindsey, pp. 185-197, Kluwer, Dordrecht, 1994.

Lamy, P., J. R. Kuhn, H. Lin, S. Koutchmy, and R. N. Smartt, No evidence of a circumsolar dust ring from infrared observations of the 1991 solar eclipse, Science, 257, 1377-1380, 1992.

Leinert, C., H. Link, and E. Pitz, Rocket photometry of the inner zodiacal light, Astron. Astrophys., 30, 411-422, 1974.

Leinert, C., I. Richter, E. Pitz, and M. Hanner, Helios zodiacal light measurements - a tabulated summary, Astron. Astrophys., 110, 355-357, 1982.

Leinert, Ch., S. Bowyer, L. K. Haikala, M. S. Hanner, M. G. Hauser, A.-Ch. Levasseur-Regourd, I. Mann, K. Mattila, W. T. Reach, W. Schlosser, H J. Staude, G. N. Toller, J. L. Weiland, J. L. Weinberg, and A. N. Witt, The 1997 reference of diffuse night sky brightness, Astron. Astrophys. Suppl. Ser. 127, 1-99, 1998

Léna, P., Y. Viala, D. Hall, and A. Soufflot, The thermal emission of the dust corona, during the eclipse of June 30, 1973, Astron. Astrophys., 37, 81-86, 1974.

Levasseur-Regourd, A. C., Optical and thermal properties of zodiacal dust, in Physics, Chemistry, and Dynamics of Interplanetary Dust, A.S.P. Conf. Ser., Vol. 104, edited by B. A. S. Gustafson and M. S. Hanner, pp. 301308, Astronomical Society of the Pacific, San Francisco, 1996.

Levasseur-Regourd, A. C., J. B. Renard, and R. Dumont, The zodiacal cloud complex, in Origin and Evolution of Interplanetary Dust, Astrophysics and Space Science Library, Vol. 173, edited by A. C. Levasseur-Regourd and H. Hasegawa, pp. 131-138, Kluwer, Dordrecht, 1991.

MacQueen, R. M., Infrared observations of the outer solar corona, Astrophys. J., 154, 1059-1076, 1968.

MacQueen, R. M. and B. W. Greeley, Solar coronal dust scattering in the infrared, Astrophys. J., 440, 361-369, 1995.

MacQueen, R. M. and O. C. St. Cyr, Sungrazing comets observed by the Solar Maximum Mission coronagraph, Icarus, 90, 96-106, 1991.

MacQueen, R. M., C. L. Ross, and T. Mattingly, Observations from space of the solar corona/inner zodiacal light, Planet. Space Sci., 21, 2173-2179, 1973.

MacQueen, R. M., K.-W. Hodapp, and D. N. B. Hall, Infrared coronal observations at the 1991 solar eclipse, in Infrared Solar Physics, IAU Symp. No. 154, edited by D. M. Rabin, J. T. Jefferies, and C. Lindsey, pp. 199-203, Kluwer, Dordrecht, 1994.

MacQueen, R. M., W. C. Davidson, and I. Mann, The role of particle size in producing the $F$-coronal scattered brightness, in Physics, Chemistry, and Dynamics of Interplanetary Dust, A.S.P. Conf. Ser., Vol. 104, edited by B. A. S. Gustafson and M. S. Hanner, pp. 349-352, Astronomical Society of the Pacific, San Francisco, 1996.

Maihara, T., K. Mizutani, N. Hiromoto, H. Takami, and H. Hasegawa, A balloon observation of the thermal radiation from the circumsolar dust cloud in the 1983 total eclipse, in Properties and Interactions of Interplanetary Dust, Astrophysics and Space Science Library, Vol. 119, edited by R. H. Giese and P. Lamy, pp. 55-58, Reidel, Dordrecht, 1985.

Mampaso, A., C. Sánchez-Magro, and J. Buitrago, Near infrared emission from the solar corona, in Sun and Planetary System, Astrophysics and Space Science Library, Vol. 96, edited by W. Fricke and G. Teleki, pp. 257-258, Reidel, Dordrecht, 1982.

Mampaso, A., C. Sánchez-Magro, M. J. Selby, and A. D. MacGregor, Infrared observations of the thermal emission from the corona, Rev. Mexicana Astron. Astrof., 8, 3-6, 1983.

Mankin, W. G., R. M. MacQueen, and R. H. Lee, The coronal radiance in the intermediate infrared, Astron. Astrophys., 31, 17-21, 1974.

Mann, I., Interplanetary dust close to the Sun, in Origin and Evolution of Interplanetary Dust, Astrophysics and Space Science Library, Vol. 173, 
edited by A. C. Levasseur-Regourd and H. Hasegawa, pp. 187-190, Kluwer, Dordrecht, 1991.

Mann, I., The solar $F$-corona: calculations of the optical and infrared brightness of circumsolar dust, Astron. Astrophys., 261, 329-335, 1992.

Mann, I., Properties and dynamics of interplanetary dust derived from near solar studies, in Meteroroids and Their Parent Bodies, edited by J. Štohl and I. P. Williams, pp. 387-390, Astronomical Inst. Slovak Acad. Sci., Bratislava, 1993a.

Mann, I., The influence of circumsolar dust on the whitelight coronastudy of the visual $F$-corona brightness, Planet. Space Sci., 41, 301-305, $1993 b$.

Mann, I., Zodiacal cloud complexes, Earth Planets Space, 50, this issue, 465-471, 1998.

Mann, I. and E. Grün, The dynamics of interplanetary dust derived from observations of the zodiacal light in relation to the dust experiments onboard Ulysses and Galileo, in Hypervelocity Impacts in Space, edited by J. A. M. McDonnell, pp. 200-204, Unit for Space Sciences, University of Kent at Canterbury, 1992.

Mann, I. and R. M. MacQueen, The solar $F$-corona at $2.12 \mu \mathrm{m}$ : calculations of near-solar dust in comparison to 1991 eclipse observations, Astron. Astrophys., 275, 293-297, 1993.

Mann, I., H. Okamoto, T. Mukai, H. Kimura, and Y. Kitada, Fractal aggregate analogues for near solar dust properties, Astron. Astrophys., 291, 1011-1018, 1994.

Matsumoto, T., M. Kawada, H. Murakami, M. Noda, S. Matsuura, M. Tanaka, and K. Narita, IRTS observation of the near-infrared spectrum of the zodiacal light, Publ. Astron. Soc. Japan, 48, L47-L51, 1996.

Michels, D. J., N. R. Sheeley, Jr., R. A. Howard, and M. J. Koomen, Observations of a comet on collision course with the sun, Science, 215, 1097-1102, 1982.

Mizutani, K., T. Maihara, N. Hiromoto, and H. Hiromoto, Near-infrared observation of the circumsolar dust emission during the 1983 solar eclipse, Nature, 312, 134-136, 1984.

Mukai, T. and S. Mukai, Temperature and motion of the grains in interplanetary space, Publ. Astron. Soc. Japan, 25, 481-488, 1973.

Mukai, T. and T. Yamamoto, A model of the circumsolar dust cloud, Publ. Astron. Soc. Japan, 31, 585-595, 1979.

Newkirk, G., Jr., R. G. Dupree, and E. J. Schmahl, Magnetic fields and the structure of the solar corona II: Observations of the 12 November 1966 solar corona, Solar Phys., 15, 15-39, 1970.

Nikolsky, G. M., S. Koutchmy, and I. A. Nesmyanovich, Relative distribution of color of June 30, 1973 solar corona, Soln. Dannye, 4, 67-70, 1983 (in Russian).

Over, J., On the vaporization of solid particles near the sun, Proc. Kon. Ned. Akad. v. Wetensch., B, 61, 74-84, 1958.
Pepin, T. J., Observations of the brightness and polarization of the outer corona during the 1966 November 12 total eclipse of the Sun, Astrophys. J., 159, 1067-1075, 1970.

Peterson, A. W., Thermal radiation from interplanetary dust, Astrophys. J., 138, 1218-1230, 1963.

Peterson, A. W., Experimental detection of thermal radiation from interplanetary dust, Astrophys. J., 148, L37-L39, 1967.

Peterson, A. W., The coronal brightness at 2.23 microns, Astrophys. J., 155, 1009-1015, 1969.

Peterson, A. W., A determination of the vaporization temperature of circumsolar dust at $4 R_{\odot}$, Bull. Am. Astr. Soc., 3, 500, 1971.

Rao, U. R., T. K. Alex, V. S. Iyengar, K. Kasturirangan, T. M. K. Marar, R. S. Mathur, and D. P. Sharma, IR observations of the solar corona-ring around the Sun? Nature, 289, 779-780, 1981.

Russell, H. N., On meteoric matter near the stars, Astrophys. J., 69, 49-71, 1929.

Saito, K., A. I. Poland, and R. H. Munro, A study of the background corona near solar minimum, Solar Phys., 55, 121-134, 1977.

Skomorovsky, V. I., S. A. Druzhinin, and R. T. Salakhutdinov, Otchet o Nabludenii Solnechnogo Zatmeniya 11 Iyulya 1991 goda s territorii Meksiki (Report on the observation of the July 11, 1991 solar eclipse in Mexico), SibIZMIR, 1992 (in Russian).

Smartt, R. N., J. Strong, W. S. Dalton, and T.-C. Li, 1973 Africa eclipse observations, NASA Report, January 1974.

Strong, J., Balloon-borne infrared coronagraph, in Telescope System for Balloon-Borne Research, NASA Technical Memorandum, edited by C. Swift, F. C. Witteborn, and A. Shipley, (NASA TM X-62397) pp. 1-6, November 1974.

Tanabé, T., F. Tsumuraya, N. Baba, M. Alvarez, M. Noguchi, and S. Isobe, Optical polarization observations of the solar corona during the total solar eclipse of 1991 July 11, Publ. Astron. Soc. Japan, 44, L221-L226, 1992.

Tollestrup, E. V., G. G. Fazio, J. Woolaway, J. Blackwell, and K. Brecher, Infrared images of the Sun during the July 11, 1991 solar eclipse, in Infrared Solar Physics, IAU Symp. No. 154, edited by D. M. Rabin, J. T. Jefferies, and C. Lindsey, pp. 179-183, Kluwer, Dordrecht, 1994.

van de Hulst, H. C., Zodiacal light in the solar corona, Astrophys. J., 105, 471-488, 1947.

Waldmeier, M., Ergebnisse der Zürcher Sonnenfinsternisexpedition 1954 VIII. Helligkeitsverteilung und Komponentenzerlegung, Z. Astrophys., 53, 81-94, 1961 (in German).

H. Kimura (e-mail: kimura@helene.mpae.gwdg.de) and I. Mann (email: mann@lindust.mpae.gwdg.de) 